\title{
Editorial
}

\section{Inflammatory role of platelets in acute coronary syndromes}

Increasing evidence shows that inflammatory mediators play a pathogenic role in atherogenesis and acute coronary syndrome. ${ }^{1}$ In particular, several reports suggest that inflammatory cytokines such as tumour necrosis factor $\alpha$ $(\mathrm{TNF} \alpha)$, interleukin (IL)-1 and various chemokines (for example, IL-8) may enhance degradation of the connective tissue matrix protein by activating matrix metalloproteinases (MMPs) and induce apoptosis of cells within the atherosclerotic lesion, promoting plaque destabilisation and rupture. ${ }^{12}$ These findings indicate a pathogenic link between persistent immune activation and plaque rupture in coronary artery disease. It is well established that platelets also contribute to the pathogenesis of acute coronary syndromes by promoting thrombus formation. However, recent studies suggest that these cells also may trigger an acute coronary event through other mechanisms, such as stimulation of an inflammatory response within the atherosclerotic plaque.

\section{Platelets as inflammatory cells}

Several lines of evidence support a role for platelets as inflammatory cells. Firstly, platelets provide a wide range of growth factors and inflammatory mediators by their release from intracellular storage organelles. Included in this group of mediators are several members of the chemokine family. In fact, platelet factor 4 and $\beta$-thromboglobulin, localised in platelet $\alpha$-granules, represented the first chemokines to be discovered. ${ }^{3}$ In recent years, a number of CCand CXC- as well as other cytokines-for example, CD40 ligand (CD40L) -have been found in these cells. ${ }^{34}$ Second, platelets do not only contain and express inflammatory mediators, but may upon activation also induce the expression of such substances (for example, $\mathrm{TNF} \alpha$ and chemokines) in monocytes/macrophages. Actually, upon activation platelets express P-selectin on their surface. Through ligation with its counterpart on monocytes/macrophages, P-selectin has the potential to enhance the activation of the transcriptional factor nuclear factor- $\kappa \mathrm{B},{ }^{5}$ a factor required for expression of chemokines, $\mathrm{TNF} \alpha$ and several other gene products playing a key role in inflammation. Notably, recent studies suggest that platelets also may modulate the function of other leucocyte subsets (for example, natural killer cells, granulocytes, and T cells) and they have been found to enhance chemotactic and adhesive properties of endothelial cells as well as IL-1 production in vascular smooth muscle cells (SMC) ${ }^{6}{ }^{7}$ Finally, platelets may not only promote an inflammatory response in leucocytes and endothelial cells, but may also themselves respond to inflammatory mediators produced by these cells. In fact, platelets have recently been found to express several chemokine receptors that upon stimulation endorse platelet activation. ${ }^{8}$ In summary, upon activation platelets may release and express inflammatory mediators, induce an inflammatory response within leucocytes, and respond with activation to several of the inflammatory mediators produced by these cells. This platelet-leucocyte cross talk seems to involve a wide range of mediators such as chemokines, adhesions molecules, reactive oxygen species (ROS), and cytokines. It is tempting to hypothesise that this inflammatory interaction between platelets and leucocytes, also involving endothelial cells, may represent a vicious circle playing a pathogenic role not only in the chronic atherosclerotic process, but also in the triggering of the acute coronary syndromes.

\section{Platelet mediated inflammation: possible role in acute coronary syndromes}

Several of the responses that have been found during animal studies and in in vitro systems when investigating the interaction between platelets, leucocytes, and endothelial cells may be relevant in acute coronary syndromes. Thus, a platelet induced upregulation of adhesion molecules and chemokines leading to increased recruitment and activation of leucocytes within the atherosclerotic plaque, the enhanced ROS production possibly promoting apoptosis of plaque stabilising cells, and the activation of MMPs could all contribute to plaque destabilisation. ${ }^{12}$ Notably, activated platelets have been shown to enhance gelatinolytic activity ${ }^{9}$ and to exert synergistic effects with leucocytes in inducing endothelial cell apoptosis in ischaemic rat models. ${ }^{10}$ Such findings further support a plaque destabilising effect of platelets. Importantly, there are also some reports suggesting that platelet related inflammation could trigger acute coronary syndromes in human. Thus, we have previously shown that platelets from patients with unstable angina are characterised by notably decreased intracellular CD40L concentrations, probably reflecting enhanced degranulation and increased release of soluble (s) CD40L in vivo. ${ }^{4}$ In this issue of Heart, Garlichs and colleagues extend these findings by demonstrating increased expression of CD40L on the platelet surface in acute coronary syndromes. ${ }^{11}$ Moreover, increased leucocyte-platelet adhesion has been reported in acute myocardial infarction, possibly contributing to the inflammatory response in this disorder. Thus, binding of activated platelets seems to induce IL- $1 \beta$ and chemokine expression in leucocytes from these patients. ${ }^{12}$ Finally, von Hundelshausen and colleagues have recently reported that the deposition of the CC-chemokine RANTES by platelets triggers shear resistant monocyte arrest on inflamed atherosclerotic endothelium, ${ }^{13}$ an event that clearly may be relevant to the triggering of acute coronary syndromes.

\section{CD40/CD40L system: key role in plaque \\ destabilisation}

Several platelet derived factors, both membrane bound (for example, P-selectin, CD40L, and integrins) and soluble (for example, chemokines and sCD40L) may be involved in the inflammatory interaction between platelets, leucocytes, and endothelial cells. Recently, particularly attention has been focused on CD40L. On platelet activation this cytokine is released in a soluble form, and expressed on the platelet surface in a membrane bound form, respectively. ${ }^{414}$ The study by Garlichs and colleagues presents new data supporting a pathogenic role for this member of the TNF superfamily in acute coronary syndromes. ${ }^{11}$ They found enhanced expression of CD40L on the platelet surface during acute myocardial infarction, and even more 
importantly, they show that enhanced CD40L expression is correlated with the need for re-angioplasty or recurrence of angina in these patients. Several lines of evidence suggest that these findings not only represent epiphenomena, but may reflect important pathogenic processes in these patients. Thus, both CD40L and its receptor CD40 is overexpressed in human and experimentally induced atherosclerotic lesions with functional receptors expressed on atheroma associated endothelial cells, SMC, macrophages, and $\mathrm{T}$ cells. ${ }^{14}$

Activation of CD40 by its ligand CD40L may have several consequences. Firstly, CD40L stimulation increases the expression of adhesion molecules, chemokines, and inflammatory cytokines leading to recruitment and activation of leucocytes within the plaque. ${ }^{414}$ Secondly, both soluble and membrane bound CD40L are potent inducers and activators of MMPs in macrophages and vascular SMC, ${ }^{14}$ and such activation may play a crucial role in undermining the integrity of tissue components in an atherosclerotic plaque. ${ }^{12}$ Finally, CD40-CD40L interaction may promote thrombotic activity by enhancing tissue factor expression in macrophages and through direct regulation of endothelium associated procoagulant activity. ${ }^{14}$ Consequently, in acute coronary syndromes activated platelets may, by providing CD40L, contribute to inflammatory reactions, MMP activation, and procoagulant activity within an atherosclerotic plaque. Such activities may in turn further enhance platelet activation and thrombus formation, possibly representing a pathogenic loop promoting plaque destabilisation and rupture during acute coronary syndromes.

\section{Therapeutic implications}

The potential inflammatory role of platelets in the pathogenesis of acute coronary syndromes may have several therapeutic implications. Firstly, platelet inhibitors such as aspirin, glycoprotein (GP) IIb/IIIa antagonists, and more recently adenosine 5'-diphosphate (ADP) receptor blockers, are already cornerstones in the treatment of acute coronary syndromes. However, aspirin in the dosage used in these patients may act as a weak inhibitor of platelet activation, and it is possible that the beneficial effect of aspirin in acute coronary syndromes is related to direct anti-inflammatory effects on leucocytes and endothelial cells. ${ }^{15}$ Moreover, although there are a few reports suggesting anti-inflammatory effects of GP IIb/IIIa inhibitors and ADP receptor blockers ${ }^{16}$ our knowledge in this area is limited. It will be of importance to examine if such agents also have the potential to inhibit platelet mediated inflammation, and if not, this may represent a major limitation of these medications. Secondly, as discussed above, recent research has delineated "new" molecules that may be key components in platelet mediated inflammation (for example, P-selectin, CD40L, and platelet related chemokines).
Targeted treatment against these molecules may represent a novel therapeutic approach in downregulation of platelet mediated inflammation and in the treatment of acute coronary syndromes. Finally, based on the inflammatory role of platelets, antiplatelet drugs may be of potential interest in the management of several other inflammatory disorders such as various autoimmune disorders and vasculitis.

PÅL AUKRUST TORGUN WEHRE

JAN KRISTIN DAMÅS LARS GULLESTAD NILS OLAV SOLUM

Section of Clinical Immunology and Infectious Diseases,

Research Institute for Internal Medicine,

Department of Cardiology,

Rikshospitalet,

$\mathrm{N}-0027$ Oslo,

Norway

Correspondence to: Dr Aukrust; pal.aukrust@rikshospitalet.no

1 Entmann ML, Ballantyne CM. Inflammation and acute coronary syndromes. Circulation 1993;88:800-3.

2 Libby P. Current concepts of the pathogenesis of the acute coronary syndromes. Circulation 2001;104:365-72.

3 Brandt E, Ludwig A, Petersen F, et al. Platelet-derived CXC chemokines.old players in new games. Immunol Rev 2000;177:204-16.

4 Aukrust P, Müller F, Ueland T, et al. Enhanced levels of soluble and membrane-bound CD40 ligand in patients with unstable angina-possible reflection of $\mathrm{T}$ lymphocyte and platelet involvement in the pathogenesis of reflection of T lymphocyte and platelet involvement in the
acute coronary syndromes. Circulation 1999;100:614-20.

5 Weyrich AS, McIntyre TM, McEver RP, et al. Monocyte tethering by $\mathrm{P}$-selectin regulates monocyte chemotactic protein- 1 and tumor necrosis factor-alpha secretion. Signal integration and NF-kappa B translocation. $\mathcal{F}$ Clin Invest 1995;95:2297-303.

6 Gawaz M, Brand K, Dickfeld T, et al. Platelets induce alterations of chemotactic and adhesive properties of endothelial cells mediated through an interleukin-1 dependent mechanism. Implication for atherogenesis. Atherosclerosis 2000;148:75-85.

7 Loppnow H, Bil R, Hirt S, et al. Platelet-derived interleukin-1 induces cytokine production, but not proliferation of human vascular smooth muscle cells. Blood 1998;91:134-41.

8 Clemetson KJ, Clemetson JM, Proudfoot AE, et al. Functional expression of CCR1, CCR3, CCR4, and CXCR4 chemokine receptors on human platelets. Blood 2000;96:4046-54.

9 Kazes I, Elalamy I, Sraer JD, et al. Platelet release of trimolecular complex components MT1-MMP/TIMP2/MMP2: involvement in MMP2 activation and platelet aggregation. Blood 2000;96:3064-9.

10 Sindram D, Porte RJ, Hoffman MR, et al. Synergism between platelets and leukocytes in inducing endothelial cell apoptosis in the cold ischemic rat liver: a Kupffer cell mediated injury. FASEB f 2001;15:1230-2.

11 Garlichs CD, Eskafi S, Raaz D, et al. Patients with acute coronary syndromes express enhanced levels of CD40 ligand/CD154 on platelets. Heart 2001;86:649-55.

12 Neumann FJ, Marx N, Gawaz M, et al. Induction of cytokine expression in leukocytes by binding of thrombin-stimulated platelets. Circulation 1997;95:2387-94.

13 von Hundelshausen P, Weber KSC, Huo Y, et al. RANTES deposition by platelets triggers monocyte arrest on inflamed and atherosclerotic endothe-

14 Schönbeck U, Libby P. The CD40/CD154 receptor/ligand dyad. Cell Mol Life Sci 2001;58:4-43.

15 Pierce JW, Read MA, Ding H, et al. Salicylates inhibit I kappa B-alpha phosphorylation, endothelial-leukocyte adhesion molecule expression, and neutrophil transmigration. F Immunol 1996;156:3961-9.

16 Neumann FJ, Zohlnhofer D, Fakhoury L, et al. Effect of glycoprotein IIb/IIIa receptor blockade on platelet-leukocyte interaction and surface expression of the leukocyte integrin Mac-1 in acute myocardial infarction. f Am Coll Cardiol 1999;34:1420-6. 